\title{
L'alliance lydo-spartiate
}

RÉsumé -. Le présent article analyse la crédibilité à reconnaître à l'alliance que le roi de Lydie Crésus aurait conclue avec la cité de Sparte. Cet examen se penche sur la consultation des oracles opérée par Crésus ainsi que le rôle joué par les offrandes du roi lydien en Grèce à la suite de celle-ci; il étudie également le statut de Sparte en tant que la plus puissante cité en Grèce vers 550. Enfin, ces quelques pages tentent de préciser la nature des liens qui unissaient la cité de Lacédémone au roi lydien en comparaison de ceux qu'il entretenait avec les Delphiens.

ABstract -. This article analyses the credibility we have to recognize to the alliance that would have been concluded between the king of Lydia Croesus and the city of Sparta. This study focuses on the oracles consultation by Croesus and the role played by the Lydian king's offerings in Greece following it. This article deals also with the status of Sparta as the most powerful city in Greece near 550 BC. Finally, those few pages try to establish the nature of the ties between Lacedaemon and the Lydian king in comparison to those he had with the Delphians.

Selon Hérodote, de retour à Sardes après avoir livré une bataille indécise en Ptérie contre Cyrus II roi des Perses, Crésus aurait projeté de convoquer ses alliés afin que ceux-ci lui amènent des troupes supplémentaires qui lui permettraient, une fois l'hiver passé, d'attaquer en force les Perses au printemps ${ }^{1}$. Cette mention fournit l'occasion à l'historien d'Halicarnasse de nous dévoiler des alliances qu'aurait conclues auparavant le roi de Lydie non seulement avec les Spartiates, mais aussi avec Amasis d'Égypte et même le roi de Babylone. L'historicité de ces accords a été diversement appréciée par l'érudition moderne, certains les reléguant dans le registre de la fiction, d'autres acceptant sans réserve leur conclusion. Dans le cadre de l'étude que nous venons d'entreprendre sur les règnes des deux derniers rois lydiens, la question ne pourra être éludée. Sans plus attendre, en quelques pages, nous nous proposons de revisiter les questions que soulèvent en particulier les accords qu'auraient conclus Lydiens et Spartiates, afin de cerner au mieux la crédibilité à leur reconnaître. Dans la foulée, nous étudierons l'épisode de la consultation des oracles grecs par Crésus et nous évaluerons l'authenticité des réponses. En prolongement, nous explorerons le statut de Sparte dans le concert des cités grecques au milieu du vi siècle. Enfin, nous analyserons le rôle joué par les offrandes du roi mermnade dans la conclusion des accords à la suite de la consultation

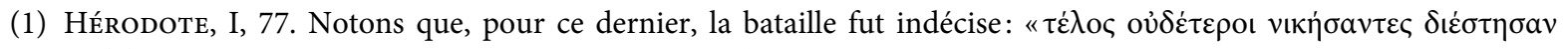

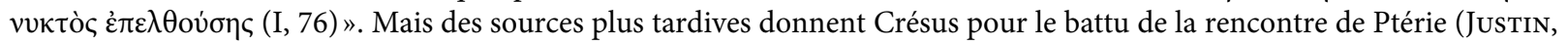
I, 7; Polyen, VII, 8). 
desdits oracles. Nous tenterons ainsi d'établir au mieux quelle était la nature des liens qui unissaient Crésus à la cité de Sparte en regard des relations du Lydien avec les Delphiens.

Le passage à considérer mérite d'être rappelé, puisqu'il va faire l'objet d'une analyse approfondie:

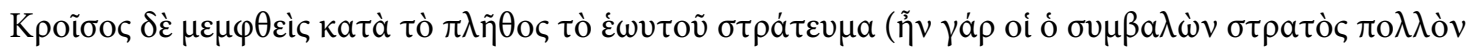

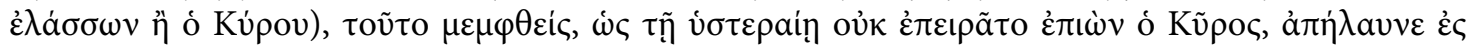

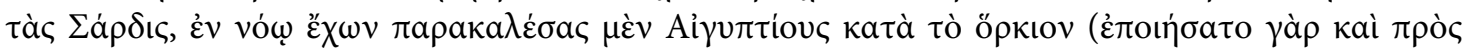

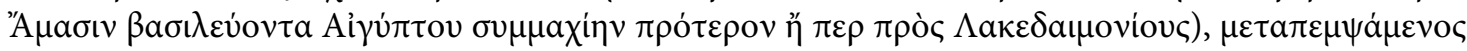

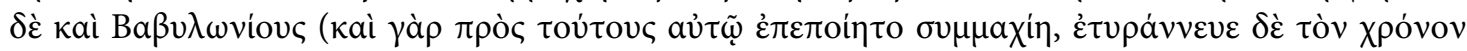

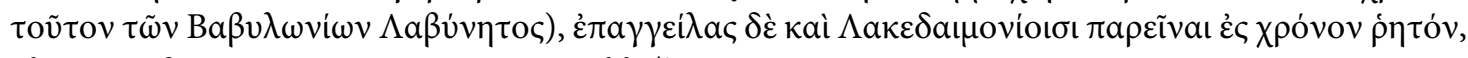

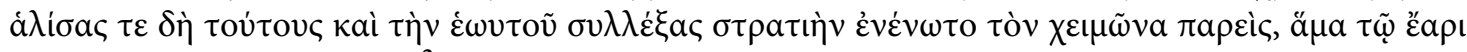

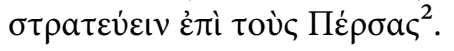

Remarquons déjà que les alliances avec Amasis et le roi de Babylone ${ }^{3}$ sont explicitées par deux incises. Dans la première, il est possible de trouver des précisions sur le traité (öркıоv) en vertu duquel Crésus appelle les Égyptiens à son secours: une alliance ( $\sigma \nu \mu \mu \alpha \chi i \eta)$ lydo-égyptienne avait été conclue avant celle avec Sparte. La seconde fournit la raison pour laquelle Crésus peut faire appel aux Babyloniens: une alliance ( $\sigma u \mu \alpha \chi i \eta)$ avait également été conclue avec le roi de Babylone, celui qu'il nomme Labynète.

Quoi qu'il en soit, toujours selon notre informateur, Crésus aurait conclu, au début de son règne, une première alliance avec les Ioniens des îles, dans la foulée d'une entrevue à Sardes avec Bias de Priène - ou Pittacos de Lesbos. Au cours de celle-ci il aurait été dissuadé d'attaquer ces Insulaires. Dès lors, plutôt que de les combattre, Crésus aurait convenu d'un traité d'amitié avec les

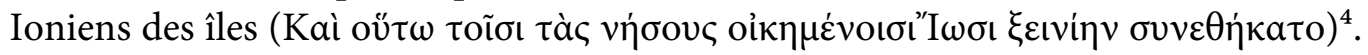

Armée de ces rappels, nous pouvons évoquer la consultation des oracles de Grèce commanditée par Crésus.

\section{LA CONSULTATION DES ORACLES}

Informé de la chute du roi des Mèdes Astyage vaincu par Cyrus, Crésus aurait décidé d'éprouver la véracité des oracles de Grèce et de Libye. Son intention aurait été, après avoir testé leur fiabilité, de leur demander par une seconde ambassade s'il devait faire la guerre aux Perses ${ }^{5}$. À la suite de ce premier examen, Crésus aurait convenu que les oracles de Delphes et d'Amphiaraos étaient les seuls

(2) «Mais Crésus, mécontent de l'insuffisance numérique de ses troupes, - car les forces qui s’étaient engagées de son côté étaient bien moins nombreuses que celles de Cyrus, - mécontent de cette disproportion, comme le lendemain Cyrus ne tentait pas d'attaque, s'en retourna à Sardes. Son intention était d'appeler au secours les Égyptiens en vertu du traité (car il avait aussi conclu une alliance avec Amasis, roi d'Égypte, avant d'en conclure avec les Lacédémoniens), de faire venir également les Babyloniens (avec eux de même il avait une alliance; le roi de Babylone était en ce temps Labynète), de mander en outre aux Lacédémoniens qu'ils eussent à se trouver à Sardes pour une date fixée; ces alliés réunis, ses propres troupes rassemblées, il avait l'intention, après avoir laissé passer l'hiver, de marcher au printemps contre les Perses». - HÉRODOTE, I, 77. Dans cet article, toutes les citations d'Hérodote proviennent de l'édition de P. E. Legrand dans la collection Budé.

(3) Hérodote mentionne un certain Labynète. Il faut reconnaître ici Nabonide, roi de Babylone de 556 à 539 avant d'être détrôné par Cyrus. Hérodote mentionnait déjà au chapitre 74 un autre Labynète qui aurait servi de médiateur dans la conclusion de la paix entre la Lydie et les Mèdes en 585. Notre auteur précise au chapitre 188 toujours de son livre I que ce dernier Labynète était le père de celui qui avait conclu l'alliance avec Crésus. Or, il s'avère qu'en 585, le roi de Babylone était Nabuchodonosor et que ce dernier n'avait aucun lien de parenté avec Nabonide. Les propos d'Hérodote sont donc confus. Voir D. Asheri et al. 2007, p. 135; voir P. E. Legrand 1932 (a), p. 183, note 1.

(4) Hérodote, I, 27. L'un et l'autre sont repris dans diverses listes nommant les Sept Sages : voir notamment les propos attribués à Socrate par PLATON, Protagoras, 342e-343b et A. Busine 2002.

(5) НÉrodote, I, 46-53. 
véridiques $(\dot{\alpha} \psi \varepsilon v \delta \varepsilon \grave{\varepsilon})$. En effet, ils avaient réussi le test auquel Crésus les avait soumis ${ }^{6}$. Hérodote relate ensuite, aux chapitres 50-52, toutes les offrandes que le roi avait fait parvenir à ces deux sanctuaires. Celles-ci semblent toujours visibles du temps de notre historien, vu sa description de celles qui étaient dédiées à Amphiaraos: «ces deux objets étaient encore de mon temps en dépôt à

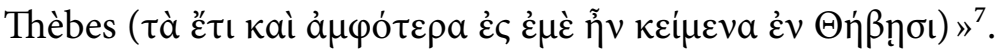

Lors de la deuxième ambassade, les Lydiens interrogèrent donc ces deux oracles véridiques ${ }^{8}$, cherchant à savoir s'il était opportun de faire la guerre aux Perses et s'il devait s'adjoindre des

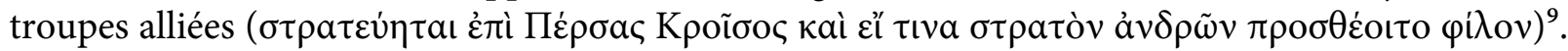

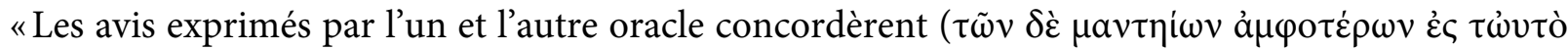
ai $\gamma v \tilde{\omega} \mu a \iota$ $\sigma v v \varepsilon \dot{\delta} \rho \alpha \mu \nu v)$ ) et ils lui conseillèrent de «s'adjoindre comme alliés ceux des Grecs qu'il

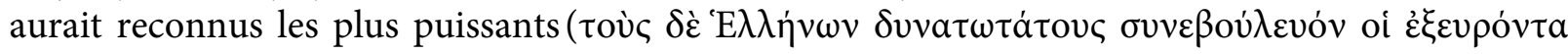
$\varphi \dot{\lambda} \lambda$ ovৎ $\pi \rho \circ \sigma \theta \dot{\varepsilon} \sigma \theta a \mathrm{l})$ », non sans lui avoir préalablement prédit qu'il «détruirait un grand empire $(\mu \varepsilon \gamma \alpha \dot{\alpha} \lambda \eta \nu$ à $\rho \chi \eta ̀ v)$ s'il faisait la guerre aux Perses $»^{10}$. À la suite de nouvelles recherches, Crésus aurait appris que le premier rang chez les Grecs était occupé par les Spartiates et les Athéniens

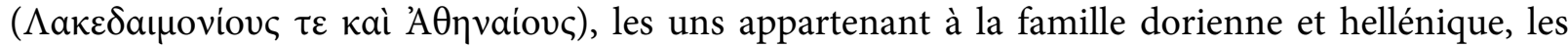
autres à la famille ionienne et pélasgique ${ }^{11}$.

C'est à partir de ces renseignements qu'Hérodote entame deux longues digressions («hors sujet», en fait anachroniques), l'une sur les habitants de Sparte, l'autre sur ceux d'Athènes. Ces développements exposent leurs origines ethniques (les premiers auraient migré, les seconds seraient installés depuis toujours). Mais pour l'essentiel, elles signalent ce qui se déroule sur le continent grec à cette époque ${ }^{12}$. Pour ce qui est de leur berceau racial, le récit d'Hérodote reflète des préoccupations de son temps (la révolution intellectuelle du $\mathrm{v}^{\mathrm{e}}$ siècle favorise les enquêtes sur les origines). En effet, comme l'a rappelé Édouard Will, «les Grecs contemporains des tyrannies archaïques [n'avaient pas] conscience de ce qui pouvait subsister de différenciation ethnique dans les cités de leur temps et surtout ils [ne le ressentaient pas] de façon affective. [...] Ce n'est qu'au $\mathrm{V}^{\mathrm{e}}$ siècle que les notions mêmes de Doriens et d'Ioniens font leur apparition dans les textes ${ }^{13}$. Néanmoins, comme l'a souligné le grand historien français, la distinction établie ici par Hérodote n'a qu'une fin généalogique. De fait, son propos ne laisse pas transparaître un quelconque antagonisme entre ces Grecs, ni ne les hiérarchise. Ce qu'Hérodote met en avant, c'est que les Doriens étaient considérés comme des Hellènes dès l'origine alors que les Athéniens, eux, étaient devenus Hellènes. Mais surtout Hérodote ne dit jamais que les Athéniens ( $a u v^{e}$ siècle) étaient perçus comme des Grecs de second rang. Ainsi, lorsqu'il parle de Doriens et d'Ioniens, l'homme

(6) HÉrodote, I, 46-49.

(7) НÉrodote, I, 52.

(8) Remarquons qu'Hérodote (I, 53) ne les mentionne pas, il déclare: «les Lydiens qui allaient conduire ces présents aux

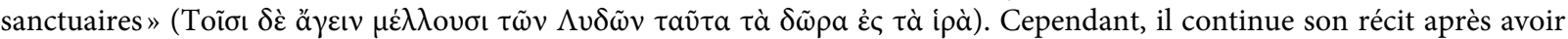
mentionné que Crésus tenait les oracles de Delphes et d'Amphiaraos comme les seuls véridiques. Ceci incline à penser que les sanctuaires mentionnés dans ce passage sont ces deux derniers.

(9) Hérodote, I, 53. Notons qu'un peu plus loin, lorsque les Lydiens s'adressent directement aux oracles, Hérodote

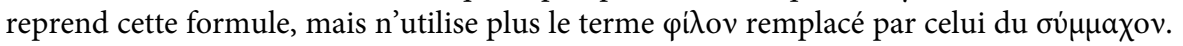

(10) Hérodote, I, 53.

(11) Hérodote, I, 56.

(12) Du chapitre 56 à 71.

(13) Au moment où les sophistes veulent affirmer l'unité de l'humanité en dépit des différences raciales. - É. WiLL 1956, p. 57 et 73. Cet antagonisme apparaît surtout lors de la guerre du Péloponnèse et s'estompera par la suite. - J. H. M. Alty 1982, p. 1-14. 
d'Halicarnasse ne fait rien de plus que de l'érudition généalogique ${ }^{14}$, en insistant simplement sur le fait que les Athéniens sont «devenus Grecs» ${ }^{15}$.

Bref, l'affirmation de l'origine pélasgique des Athéniens - et donc de leur autochtonie - met surtout en relief les préoccupations du $\mathrm{v}^{\mathrm{e}}$ siècle, dominées par la lutte idéologique entre Athènes et Sparte. L'ensemble des récits mythiques relatifs à l'autochtonie revendiquée par les Athéniens sert à légitimer leur préséance, voire leur hégémonie, et donne également un fondement à leur conception de la citoyenneté: les Athéniens sont de vrais citoyens. En effet, ils proviennent du génos originel (celui d'Érichthonios), contrairement aux Spartiates qui ne connaissent pas la démocratie ${ }^{16}$.

Revenons-en à l'alliance lydo-spartiate, mais, lorsque le moment viendra d'évaluer l'historicité de l'ensemble de l'épisode, on ne pourra oublier que ces propos d'Hérodote semblent peu en rapport avec la question posée par Crésus.

\section{L'ALLIANCE AVEC LES SPARTIATES}

Selon Hérodote, une fois au courant de la supériorité des Lacédémoniens sur les Athéniens, Crésus se serait tourné du côté des Spartiates afin de se lier à eux ${ }^{17}$. Le roi de Lydie leur aurait envoyé des messagers (å $\gamma \gamma \varepsilon \dot{\varepsilon} \lambda o v \varsigma)$ porteurs de cadeaux ( $\delta \tilde{\omega} \rho \alpha)$ et d'une annonce: Crésus les avait

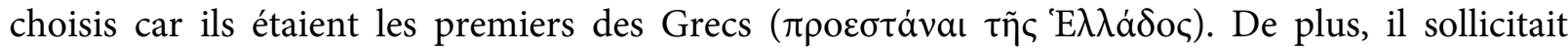
leur amitié de même que leur alliance (

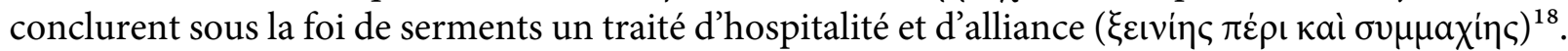
Les Lacédémoniens y auraient d'autant plus consenti qu'un contact ancien avait déjà été établi avec Crésus: plus tôt, le Lydien leur avait fourni gracieusement l'or qu'ils avaient utilisé pour ériger une statue en l'honneur d'Apollon, une œuvre visible du temps d'Hérodote sur le mont Thornax qui domine Sparte ${ }^{19}$. De plus, ils se disaient flattés d'avoir été préférés aux autres Grecs. Les Spartiates se déclarèrent disposés à répondre à son appel et lui envoyèrent alors un cratère de bronze. Mais celui-ci ne parvint jamais à Sardes pour des motifs contradictoires qu'Hérodote se plaît à rapporter par le menu ${ }^{20}$.

Un tel échange de cadeaux opéré entre Crésus et les Spartiates suggère qu'il s'agit d'une relation de réciprocité. Comme le souligne Hans Van Wees ${ }^{21}$, la fonction fondamentale de ce type de relation vise à l'établissement et l'entretien de relations amicales, les échanges constituant un outil de cohésion sociale. Il fait aussi observer qu'afin de nourrir cette relation, rien n'oblige à faire parvenir le contre-don dans la foulée du "premier» cadeau réceptionné ${ }^{22}$. De fait, dans le cas qui nous occupe, si Hérodote déclare que Crésus avait déjà envoyé de l'or à Sparte, rien ne permet de penser que le Lydien avait reçu quoi que ce soit en retour. Par ailleurs, cet échange s'apparente du

(14) É. Will 1956, p. 61-65.

(15) R. Thomas 2001, p. 222-225.

(16) N. Loraux 1979, p. 3 et 11; N. Loraux 1996, p. 37-42 et 49-59. Voir également J. M. Hall 1999, p. 50-53; R. Thomas 2001, p. 222-225.

(17) En effet, Athènes était alors sous la tyrannie des Pisistratides et Sparte, qui était «heureuse dans les autres guerres», venait de vaincre les Tégéates. - HÉrodote, I, 56-69.

(18) Hérodote, I, 69.

(19) Selon Pausanias, qui écrit six siècles plus tard, l'or envoyé par Crésus servit pour la statue de l'Apollon à Amyclées et non pas pour celle qui se trouvait sur le Thornax. - Pausanias, III, 10, 8. Nous y reviendrons ci-après.

(20) HÉrodote, I, 69-70.

(21) H. Van Wees 1998, p. 13-49.

(22) Van Wees s'appuie ici sur les travaux des sociologues Pierre Bourdieu et Alvin Wald Gouldner. Sur cette pratique d'échange de cadeaux au Proche-Orient ancien: M. Liverani 2003, p. 123-125. 
coup à une forme de dette que l'on contracte et que valide le délai de réaction : celui qui accepte le cadeau proposé se sent dans l'obligation de faire de même, en offrant pour bien faire un présent d'une valeur plus grande que celui qui a été reçu. Cet effet d'éternel déséquilibre fait en sorte que chaque partie est toujours endettée vis-à-vis de l'autre ${ }^{23}$. Dans cette perspective, les Lacédémoniens se seraient sentis redevables après avoir reçu et accepté naguère l'or commandé à Crésus sans contrepartie immédiate. Dès lors, ils lui auraient accordé leur amitié et leur alliance, le tout scellé par l'envoi d'un cratère de bronze ${ }^{24}$.

\section{QUELLE AUTHENTICITÉ ACCORDER AUX ORACLES DE LA PYTHIE ET À LA PRÉTENDUE HÉGÉMONIE SPARTIATE}

En dépit des nombreux détails fournis par Hérodote, la réalité historique de cette première alliance conclue par Crésus a provoqué incrédulité et scepticisme. En effet, tout en précisant que le fondement de cette alliance était discuté depuis longtemps, Roland Crahay a mis en doute l'authenticité des oracles. Ainsi, dans son étude sur La littérature oraculaire chez Hérodote, il juge apocryphes ${ }^{25}$ les réponses de la Pythie qui exhortait Crésus à s'adjoindre les plus puissants des Grecs. L'un des arguments avancés est que, selon lui, cette suprématie lacédémonienne dont Crésus aurait été informé, n'était pas d'actualité du temps du roi mermnade: elle daterait seulement de la fin $\mathrm{du} \mathrm{VI}^{\mathrm{e}}$ siècle - soit plusieurs décennies plus tard. Crahay est prêt à convenir que Sparte surpassait la puissance athénienne vers 550, mais il estime que, plus tôt, les Lacédémoniens ne passaient pas (encore) pour les plus puissants de tous les Grecs du continent ${ }^{26}$.

Cette position souffre la critique: même si les Spartiates n'étaient pas encore vraiment les premiers de tous les Grecs, Lacédémone jouissait déjà d'une puissance très redoutable aux alentours des années 550. À cette époque, elle avait entrepris une politique d'alliances avec ses cités voisines. De plus, elle se posait comme libératrice des tyrans et comme rempart contre les agressions. C'est d'ailleurs ainsi qu'elle avait réussi à placer sous son aile des cités loin de lui être limitrophes telles que Sicyone, Élis, l'Arcadie, Corinthe, Mégare, et peut-être Phlionte et Cléones. De plus - et surtout, en prenant le dessus sur Argos à l'issue de la bataille pour le contrôle de Thyréa, une joute que la tradition place vers $547^{27}$, elle s'était imposée comme la plus puissante communauté du Péloponnèse $^{28}$.

Un deuxième élément suscitant la méfiance a été relevé par Roland Crahay: selon lui, c'est seulement lorsque Cléomène fut capable d'intriguer à Delphes (il usait alors de son influence pour «dicter» ses oracles à la Pythie $)^{29}$, que Sparte fut apte à se faire patronner par Apollon. Ainsi, elle

(23) H. Van Wees 1998, p. 25-26. Voir également au départ M. Mauss 1923-1924.

(24) Que le cratère soit parvenu ou non à Sardes, Crésus s'est cru en droit d'espérer un geste prochain tout comme de prétendre en retour à une aide militaire des Lacédémoniens.

(25) R. Crahay 1956, p. 198-199. Pour R. Crahay, cet oracle n'aurait été rendu que par la Pythie, car le thème de la véracité de l'oracle d'Amphiaraos (qui, selon Hérodote, était tenu également par Crésus comme véridique) aurait été inséré après coup; son affirmation est développée aux pages 195 à 197 de son ouvrage.

(26) Idem, p. 157.

(27) Hérodote, I, 82. Selon Hérodote, cette «bataille des champions» (Spartiates et Argiens s'étaient accordés à faire se mesurer chacun leurs trois cents meilleurs hommes) pour le contrôle de la Thyréatide avait eu lieu au même moment

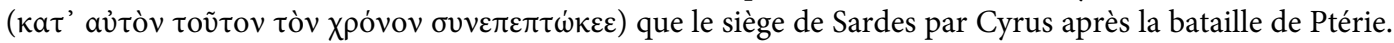

(28) N. G. L. Hammond 1982, p. 356.

(29) Cléomène réussit à obténir le concours de la Pythie lorsque celle-ci déclara que son rival Démarate, deuxième roi de Sparte, n'était pas le fils d'Ariston, le roi précédent. - Hérodote, VI, 66. Crahay ajoute que Cléomène aurait usé de son influence à Delphes afin de mettre en circulation des oracles déclarant que la dynastie des Agiades avait plus de prestige que 
aurait été reconnue comme abritant les Grecs les plus puissants de cette époque. En effet, ce n'est qu'à partir du règne de Cléomène - menant une politique agressive efficace, que Sparte aurait été en mesure de se faire entendre dansla politique intérieure en Grèce. Pour Crahay, la raison en est simple: elle se retrouvait à la tête de la Ligue du Péloponnèse qui rassemblait en son sein presque toutes les cités de l'«île de Pélops» ainsi que Mégare et Égine (chaque membre de cette ligue jurant fidélité à Sparte reconnue comme son $\dot{\eta} \gamma \varepsilon \mu \omega \omega v)$. Mieux encore, Cléomène allait jusqu’à interférer dans les politiques béotienne et athénienne ${ }^{30}$. En définitive, l'affirmation qui faisait de Sparte la cité la plus puissante parmi les cités grecques aurait eu plus de crédibilité si elle avait renvoyé à une situation postérieure d'un quart de siècle. Cet érudit fait également valoir que les propos d'Hérodote - mettant Athènes et Sparte sur un pied d'égalité lorsque Crésus cherche à connaître l'identité des Grecs les plus puissants - ont tout l'air de n'être qu'un anachronisme jouant sur des prétentions athéniennes postérieures à $480^{31}$. Ainsi, pour qui suit l'argumentaire de R. Crahay, on peut conclure au caractère imaginaire de l'alliance entre Crésus et Sparte, y voir une légende créée à l'instigation de Cléomène afin de rehausser le prestige de sa cité... et, par la même occasion, le sien.

Vincenzo La Bua rejette au contraire les doutes semés sur cette alliance. Pour sa part, il insiste sur la réalité historique qu'il convient de lui reconnaître. Ainsi, dans un article, Gli Ioni e il conflitto lidio - persiano ${ }^{32}$, il souligne que lorsque les Ioniens (et les Éoliens) firent face à Harpage après la chute de Crésus (en 547) (33 $^{3}$, ce sont les Spartiates que sollicitèrent les Ioniens. De même, il rappelle que seuls les Milésiens s'abstinrent du fait de leur liaison avec Cyrus par un serment (öpкıоv) qui remplaçait celui qui avait été échangé avec Crésus : «Les Ioniens [...] se réunirent tous au Panionion, excepté les Milésiens; car avec ceux-là seuls Cyrus avait conclu un traité aux mêmes conditions que le Lydien; les autres, d'un commun accord, décidèrent d'envoyer des députés à Sparte, pour

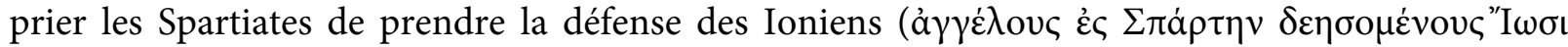
$\tau(\mu \omega \rho \varepsilon ́ \varepsilon เ v){ }^{34}$. Cependant, poursuit Hérodote après un long développement sur les Ioniens d'Asie, les Spartiates refusèrent catégoriquement d'accéder à la demande des délégués ioniens et éoliens formulée au nom de tous par Pythermos de Phocée. L'historien précise qu'ils envoyèrent tout de même une pentécontère pour y voir plus clair. Une fois arrivé à Phocée, l'équipage délégua à Sardes un certain Lacrinès, le plus estimé d'entre eux, afin d'informer Cyrus, en termes forts, sur l'interdiction lancée par les Lacédémoniens de dévaster la terre grecque. En effet, ils ne verraient pas un tel geste d'un bon œil. Et Hérodote de s'attarder malicieusement sur la fin de non-recevoir d'un Cyrus méprisant les agoras et ses pratiques propres aux Grecs ${ }^{35}$.

Il s'agit encore une fois ici d'une mention très claire de la puissance spartiate, puisque les Ioniens implorent sans hésitation l'aide de cette seule cité grecque (du continent) ${ }^{36}$. Quoi qu'il en soit, ces mentions de la suprématie spartiate confortaient R. Crahay dans l'idée qu'Hérodote aurait reçu ces

celle des Eurypontides et qu'il pouvait vaincre Argos sans la détruire, et qu'il mena une campagne pour discréditer Milet, etc. - R. Crahay 1956, p. 161-182.

(30) M. I. Finley 1982, p. 114 ; L. H. JefFery 1988, p. 353-361.

(31) R. Crahay 1956, p. 198-199. En effet, Hérodote déclare (I, 56) que les Athéniens et les Spartiates sont ex aequo ( $\tau \varepsilon$ кai), ils occupent tous les deux le premier rang. Ceci est assez intriguant surtout dans l'idée d'une hégémonie. C'est pourquoi on est en droit de penser que cela n'est peut-être qu'un ajout, de la part d'Hérodote, laissant transparaître les prétentions athéniennes en vigueur au $\mathrm{V}^{\mathrm{e}}$ siècle, Athènes voulant s'afficher comme l'égale de Sparte dès le milieu du $\mathrm{VI}^{\mathrm{e}}$ siècle.

(32) V. La Bua 1977.

(33) Donc après la «bataille des champions» entre Lacédémone et Argos (ca. 547, si celle-ci est contemporaine de la chute de Sardes comme l'avance Hérodote).

(34) Hérodote, I, 141.

(35) HÉrodote, I, 152-153.

(36) R. Crahay 1956, p. 157. 
informations d'une source favorable à Lacédémone lors de sa visite au sanctuaire de Delphes ${ }^{37}$. Il est vrai qu'Hérodote doit avoir séjourné en Grèce continentale aux alentours des années 447 à $444 / 3$ (date de son départ pour Thourioi) ${ }^{38}$ et que Sparte a pu peser sur le sanctuaire phocidien tout au long du $\mathrm{v}^{\mathrm{e}}$ siècle - en particulier après la bataille de Coronée de $447^{39}$. Par conséquent, on ne peut exclure que l'historien ait visité un sanctuaire d'Apollon sous forte influence spartiate, où les Lacédémoniens étaient libres de faire circuler des récits et des oracles fictifs ou non destinés à rehausser leur prestige ${ }^{40}$.

En revanche, afin de conforter sa thèse d'une hégémonie spartiate plus précoce, V.LaBua invoque un passage du logos samien d'Hérodote ${ }^{41}$, qui traite de la fuite des Samiens chassés par Polycrate et venus demander de l'aide à Sparte. Cette dernière, selon les Samiens, aurait alors attaqué Samos pour deux raisons: d'une part, pour s'acquitter en retour du secours porté plus tôt à Lacédémone contre les Messéniens par la flotte samienne; de l'autre pour venger les vols commis par des Samiens du fameux cratère offert par Sparte à Crésus, ainsi que du corselet de grande valeur expédié par Amasis aux Spartiates ${ }^{42}$. Sur cette base, La Bua conclut à l'existence d'une réelle alliance car, estime-t-il, dans le cas contraire, l'épisode du cratère volé aurait été inutile ${ }^{43}$. L'incident donne également l'occasion à cet érudit de souligner le rôle joué par Amasis dans la conclusion de l'alliance lydo-spartiate, puisque selon V.La Bua, le pharaon avait incité les Lacédémoniens à accepter l'accord ${ }^{44}$.

Quoi qu'il en ait été, comme se sont appliqués à le montrer R. Crahay et, avant lui, W.H. Parke, l'ensemble des oracles rendus par la Pythie à Crésus peuvent être considérés comme des oracles produits a posteriori, ou de véritables faux historiques (même s'ils sont sans doute nourris de souvenirs plus ou moins précis) ${ }^{45}$. Aussi, pour tenter d'y voir plus clair, est-il opportun de s'interroger sur la raison de l'envoi d'offrandes par Crésus en Grèce et notamment au sanctuaire de

(37) Idem, p. 156. L'ensemble des mentions de la suprématie spartiate chez Hérodote se retrouve aux chapitres 157 à 159 de son livre I. Cependant, on pourrait tout aussi bien envisager qu'Hérodote ait puisé ses informations concernant Crésus dans une, voire plusieurs tragédies du $\mathrm{VI}^{\mathrm{e}}$ siècle traitant de la famille des Mermnades, aujourd'hui perdues. Voir entre autres à ce sujet: S. SAÏD 2002; C. SEgal 1971; O. TAPLin 1997.

(38) Voir le volume introductif de Legrand dans la collection Budé: P. E. Legrand (b) 1932, p. 29-32; D. Lenfant 2011, p. 215.

(39) «Dans les années postérieures à Coronée, avec l'élimination définitive des Athéniens de la Phocide et de la Béotie, la domination de Sparte sur Delphes fut incontestée». - M. Sordi 1957, p. 64; mais aussi G. DAux 1957, p. 114-116; et surtout P. SÁnCHEz 2001, p. 114.

(40) La suprématie spartiate sur le sanctuaire de Delphes à cette époque ne remet pas en cause l'engagement de Lacédémone en Ionie, lors des campagnes d'Harpage, même s'il ne consistait qu'en l'envoi d'une pentécontère. Érétrie fera de même lors de la révolte d'Ionie une cinquantaine d'années plus tard.

(41) Hérodote, III, 39-60.

(42) Hérodote, III, 46-47. Hérodote ne mentionne qu'une seule fois le vol de ce corselet égyptien. Celui-ci aurait été de la même facture que celui que le pharaon aurait offert à l'Athéna de Lindos (II, 182). Le corselet destiné aux Spartiates aurait été volé par les Samiens juste un an avant le vol du cratère. La version du vol du cratère est, pour Hérodote, la version spartiate, car la version samienne veut que les Lacédémoniens aient revendu le cratère à Samos après avoir appris la chute de Sardes (I, 70). Donc les Samiens auraient volé le corselet un an tout au plus avant le cratère. Mais plus loin, Hérodote établit une datation relative de ce vol en déclarant qu'il aurait eu lieu à peu près au même moment que le règne de Cypsélos de Corinthe et d'Alyatte de Lydie, le père de Crésus (III, 48). Hérodote s'emmêle donc dans ses datations et justifications.

(43) Cependant, cela indique tout du moins qu'il existait aussi des liens entre Amasis et les Spartiates. Peut-être les liens ont-ils été tissés après la victoire de Sparte contre Argos (contemporaine selon Hérodote de la chute de Sardes), qui a fait de Lacédémone la plus puissante cité du Péloponnèse, voire du monde grec au vu de la place exceptionnelle des Argiens dans les productions épiques.

(44) V. La BuA 1977, p. 40-43.

(45) Le rôle d'Amphiaraos sera discuté ci-après. R. Crahay 1956, p. 182-207 ; H. W. Parke et D. E. W. Wormell 1956, p. 126-140. 
Delphes. En effet, la présence des libéralités du roi lydien au sanctuaire est une donnée historique qu'on ne peut mettre en doute ${ }^{46}$.

\section{LES LIENS ENTRE LES OFFRANDES DE CRÉSUS ET LES ACCORDS LYDO-SPARTIATES}

\section{Au Thornax}

Selon Hérodote ${ }^{47}$, c'est après avoir eu connaissance de la chute de son beau-frère Astyage (ca. 550) que Crésus se tourna vers la Grèce continentale. Cette orientation s'explique peut-être par l'espoir de Crésus d'y trouver un éventuel soutien face à une menace perse toujours plus inquiétante. Il aurait alors envoyé de nombreux dons au sanctuaire de Delphes. Mais, ce faisant, il poursuivait aussi la politique de son père Alyatte ${ }^{48}$ avec l'idée plus précise de flatter les Grecs et de ranger l'oracle de son côté. De la sorte, il espérait que ce dernier jouerait en sa faveur dans la conclusion d'une alliance. Comme le firent les Alcméonides, Crésus aurait pu intriguer auprès de la Pythie afin que Delphes invite les Lacédémoniens à accepter son alliance ${ }^{49}$.

Un fragment de Théopompe de Chios conservé chez Athénée de Naucratis ${ }^{50}$ témoigne de cette politique: "Les Lacédémoniens, voulant dorer ( $\chi \rho v \sigma \tilde{\omega} \sigma a \mathrm{l})$ la statue d'Apollon d'Amyclées, ne trouvèrent pas d'or en Grèce. Ils envoyèrent consulter l'oracle [de Delphes], pour savoir où ils pourraient en acheter. Il leur répondit : chez Crésus, roi de Lydie. Ils envoyèrent les députés chez lui,

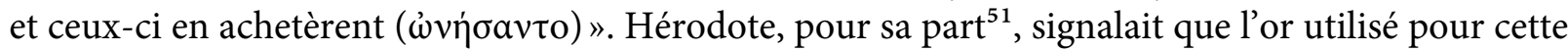
statue d'Apollon avait été offert par Crésus et que l'objet se trouvait au Thornax. Pausanias, quant à lui ${ }^{52}$, déclare - mais beaucoup plus tard, que les statues d'Apollon du Thornax et d'Amyclées étaient presque identiques. Il ajoute que celle du Thornax était réalisée selon le modèle d'Amyclées, mais que les Spartiates honoraient davantage cette dernière. En effet, pour eux, celle-ci avait été réalisée avec l'or de Crésus, qui, au départ, devait servir à décorer la statue du Thornax.

Sans qu'il soit possible de les étayer, deux premières explications relatives à l'existence de ces deux statues sont envisageables: la première consiste à penser que Crésus avait fourni de l'or ${ }^{53}$ pour la confection d'une unique statue. Placée dans un premier temps au Thornax, elle aurait été rapidement déplacée à Amyclées où elle était toujours visible au $\mathrm{II}^{\mathrm{e}} \mathrm{s}$. de notre ère ${ }^{54}$. Dans ce cas,

(46) H. W. Parke et D. E. W. Wormell 1956, p. 131.

(47) Hérodote, I, 46.

(48) Hérodote, I, 25. Cependant, Alyatte n'avait pas encore d'inquiétude de ce genre. La raison de l'envoi de ses offrandes doit donc être en partie à rechercher ailleurs, sans doute des relations de bon voisinage comme invite à la penser la densité des échanges entre les deux rives de l’Égée.

(49) Не́rodote, V, 63. Sur le rôle joué par Delphes dans la conclusion de l'alliance entre Crésus et Sparte et notamment sur la présence du $\pi \varepsilon \rho \iota \rho \rho \nu \tau \eta \dot{\rho}$

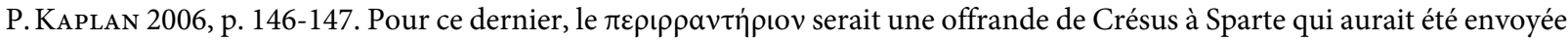
par la suite à Delphes; ou un cadeau de Crésus à Delphes, mais au nom des Lacédémoniens.

(50) ThÉOMPOMPe DE Chios, FGrH 115 F 193 J.

(51) Hérodote, I, 69.

(52) Pausanias, III, 10, 8. Pausanias a vu de ses propres yeux la statue de l'Apollon d'Amyclées: il le dit lui-même

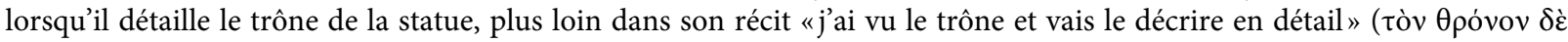

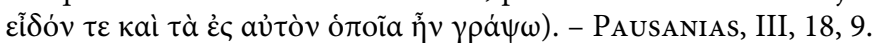

(53) Pour Hérodote et Pausanias, l’or aurait été donné par Crésus aux Spartiates; selon Théopompe, le Lydien le leur aurait vendu.

(54) Cette statue d'Apollon d'Amyclées est présente sur les monnaies de Lacédémone depuis le iII siècle av. J.-C. jusqu’à l'époque impériale romaine. Cet Apollon était représenté «casqué, debout à droite, la main droite levée brandissant la lance, le bras gauche plié à angle droit, un arc à la main gauche; le corps est dissimulé par une gaine qui tombe jusqu'aux pieds en s'élargissant légèrement vers la bas; sur la gaine se détachent un aplustre surmonté d'un coq; à droite, tout contre la statue, un bouc; à gauche, une couronne». - L. LACRoIx 1949, p. 54-58. 
Pausanias aurait été induit en erreur par ses témoins ou ses lectures. Selon la seconde explication, plus faible, l'or lydien aurait permis, d'emblée la réalisation de deux statues qui auraient ainsi encadré la cité. Hérodote ne connaissait que l'exemplaire du Thornax, Théopompe celui d'Amyclées ${ }^{55}$. On sait en tout cas que les Spartiates vouaient un culte fervent à Apollon d'Amyclées ${ }^{56}$ et faire de cette statue celle qui avait été fabriquée grâce à l'or offert par Crésus rehaussait son prestige. Nicolas Richer vient de proposer une autre hypothèse: puisque Pausanias déclare que l'or du roi lydien avait été détourné pour la réalisation de l'Apollon d'Amyclées, "ce détournement pourrait suggérer que - peut-être pour obtenir l'aide de la cité - Crésus avait envoyé l'or à Sparte pour l'Apollon du Thornax, immédiatement avant de perdre le pouvoir, mais que les Spartiates utilisèrent à leur guise l'or envoyé, une fois Crésus abattu par Cyrus ${ }^{57}$.

Cependant, remarquons que seul Pausanias qualifie de «Pythéen » l'Apollon du Thornax ${ }^{58}$, alors que ni Théopompe de Chios, ni Hérodote ne donnent d'épiclèse à cette statue (å $\gamma \alpha \lambda \mu \alpha)$. Or, le culte d'Apollon Pythéen était d'origine argienne: le dieu était notamment vénéré dans le sanctuaire de la ville d'Argos, d'Asiné, mais aussi en Cynourie (région de Thyréa) ${ }^{59}$. Dès lors, il est possible que ce soit à la suite de la "bataille des champions» et de la victoire spartiate sur Argos que le culte d'Apollon Pythéen a été installé sur l'agora ${ }^{60}$, et a fortiori sur le Thornax. Située sur la route reliant Argos à Lacédémone, la statue d'Apollon du Thornax commémorait en bonne place (tout comme la statue d'Apollon Pythéen sur l'agora) ${ }^{61}$ la conquête de la Thyréatide ${ }^{62}$.

Sur la base de ces nouvelles constatations, deux hypothèses inédites peuvent être avancées quant à l'envoi d'or par Crésus à Lacédémone: selon l'une, le Mermnade aurait envoyé de l'or pour une statue d'Apollon dressée au Thornax (qui protégeait la cité des dangers venus du nord, territoire sous contrôle argien). À la suite de la victoire de Thyréa, cet å $\gamma \alpha \lambda \mu \alpha$ du Thornax aurait été déplacé à Amyclées et remplacé par une (deuxième) statue d'Apollon pythaeus afin de célébrer la prise de la Thyréatide. Selon l'autre hypothèse, ce serait à la suite de leur victoire sur les Argiens que les Spartiates (qui passaient dès lors pour les plus puissants des Grecs) se seraient fournis en or auprès de Crésus afin de dorer l'Apollon du Thornax qui célébrait la prise de la Thyréatide. Cependant, pour une raison inconnue, cet or aurait été détourné au profit de la statue d'Amyclées. Cette dernière hypothèse impliquerait d'avancer la victoire pour le contrôle de Thyréa de quelques années avant 547 , date traditionnelle retenue ${ }^{63}$.

\section{À l'Amphiareion de Thèbes}

Des déductions similaires sont à envisager au sujet des offrandes que Crésus aurait faites à Amphiaraos ${ }^{64}$. Si les oracles sont apocryphes, nous pouvons aussi nous interroger sur les offrandes

(55) Le Thornax se situant au nord de Lacédémone, Amyclées au sud. - N. Richer 1994, p. 85; N. Richer 2012, p. 230. Mais ce choix est en contradiction avec les propos d'Hérodote qui ne mentionne qu'une statue qu'il situe sur le Thornax (I, 69).

(56) Pausanias, III, 10, 8. Voir également M. Nafissi 2009, p. 119.

(57) N. Richer 2012, p. 351.

(58) Pausanias, III, 10, 8.

(59) M. Piérart 1990; N. Deshours 1999, p. 471-473. L'épiclèse "pythéen» renvoie à Pythaeus, fils d'Apollon venu de Delphes, qui aurait construit le temple d'Apollon à Argos. - Pausanias, II, 24, 1-2.

(60) F. De Polignac 1995, p. 72-73.

(61) Idem, p. 73.

(62) Cette victoire sur Argos fut d'abord célébrée lors de la fête des Parparonia. Celle-ci fut intégrée aux Gymnopédies après 370/369 à la suite de la perte de la Thyréatide, face aux Thébains, qui empêchait une célébration sur place. - N. RICHER 2012, p. 227 n. 12.

(63) Sur la seule base des propos d'Hérodote (I, 82), qui établit un synchronisme entre la «bataille des champions» et la chute de Sardes.

(64) HÉrodote, I, 52. 
de Crésus à Thèbes. Hérodote déclare que le bouclier et la lance, tous deux en or et consacrés à Amphiaraos (dont il avait appris le mérite et le malheur), se trouvaient de son temps dans le temple

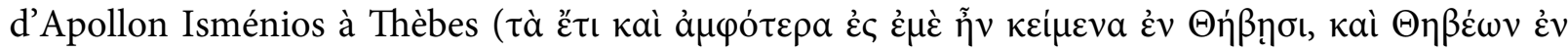

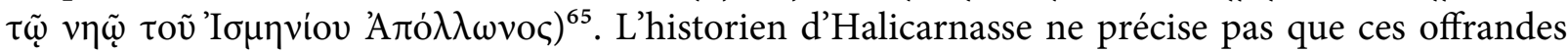
étaient au départ entreposées dans l'Amphiareion, ce qui n'invite guère à poser l'hypothèse d'un déplacement ou d'un transfert de ces offrandes. Notons d'emblée, comme le précise Pierre Sineux, après un long argumentaire, qu'il s'agit de l'Amphiareion de Thèbes et non de celui d'Oropos. En effet, l'oracle se trouvait à l'origine à Thèbes et il n'a été dédoublé à Oropos que dans la deuxième moitié du $\mathrm{v}^{\mathrm{e}}$ siècle ( $\left.\mathrm{ca} .420\right)$, lorsque la zone était sous contrôle athénien ${ }^{66}$.

Pour Pierre Sineux, ce bouclier et cette lance se trouvaient donc tout d'abord dans l'Amphiareion thébain avant d'être déplacés dans le temple d'Apollon Isménios. Ce choix du temple d'Apollon n'aurait pas été anodin puisqu'il était lui aussi le siège d'un oracle, alors qu'Amphiaraos passait aussi pour le protégé d'Apollon ${ }^{67}$. De plus, l'oracle d'Apollon Isménios pratiquait l'empyromancie; or Amphiaraos avait pratiqué au moins une fois de son vivant cette technique ${ }^{68}$. Le souvenir de cette faculté du devin a donc pu intervenir lors du transfert des offrandes de Crésus ${ }^{69}$. Cela dit, la mention du recours d'Amphiaraos à l'empyromantie ne se trouve mentionnée que chez Euripide, soit bien après le séjour d'Hérodote en Grèce continentale (ca.447-443). Euripide a donc pu faire de cette technique une pratique du héros argien pour deux raisons : à la fois parce qu'Apollon Isménios s'exprimait à travers des techniques empyromantiques, et parce qu'Amphiaraos était son protégé. Si tel est le cas, au contraire de ce que défend P. Sineux, le transfert des offrandes de Crésus n'aurait aucun lien avec cette aptitude du devin $\operatorname{argien}^{70}$.

Revenons au geste de Crésus vis-à-vis de l'Amphiareion. Ici aussi, il est probable que l'offrande du roi lydien soit une nouvelle marque de flatterie. Sans doute considérait-il les Thébains comme des partenaires potentiels. Thèbes était une autre cité très florissante du vi ${ }^{\mathrm{e}}$ siècle, au cœur du pays béotien puisqu'à la tête de la première Confédération béotienne. Elle avait également remporté quelques beaux succès militaires durant ce siècle. Enfin, elle se revendiquait à travers le cycle thébain d'un passé mythique aussi prestigieux que celui d'Argos qui venait pourtant de s'incliner devant les Spartiates ${ }^{71}$. Crésus aurait donc flatté la vieille Cité aux sept portes afin que celle-ci accepte de se lier à lui: sa place de choix dans la poésie épique et dans la tragédie attique est incontestable. Elle devait, comme pour Argos, assurer son rayonnement au loin. Ainsi s'expliquerait - tout comme à Delphes et au Thornax - la présence d'offrandes du Lydien à Thèbes. Quant à l'endroit

(65) Hérodote, I, 52

(66) L'amphiareion de Thèbes se trouvait probablement à Cnopia, entre Thèbes et Ptonia (PAusAnIAs, IX, 19, 4, et Strabon, IX, 2, 10-11). Les Athéniens ont installé le culte d'Amphiaraos à Oropos lors de la guerre d'Archidamos, Athènes cherchait à se réapproprier en partie la légende des Sept contre Thèbes. Le devin Amphiaraos, englouti en terre thébaine, se voit consacrer un culte à Oropos (alors terre athénienne). Cela contribue à signifier la mainmise des Athéniens sur Oropos face aux Thébains. De plus, cette installation du culte à Oropos s'inscrit dans une politique athénienne où l'alliance avec Argos est recherchée. - P. Sineux 2007, p. 65-79 et 95-97.

(67) Dans les Sept contre Thèbes d'Eschyle, Apollon fait des promesses à son favori (vers 618); dans l'Odyssée, il est le «favori d'Apollon» (chant XV, vers 245-246); pour Euripide, il pratique «l'art de Phoïbos» (Hypsipyle, fr. 60, I, vers 58-59).

(68) EuRIPIDE, Hypsipyle, fr. 60.

(69) P. Sineux 2007, p. 195-196.

(70) De plus, Hypsipyle d’Euripide aurait été écrite entre 410 et 407 (O. TAPLIN 2007, p. 211.), époque où le sanctuaire d'Oropos (dont l'oracle du héros argien venait d'être installé depuis peu) sous contrôle athénien était retombé sous l'autorité des Béotiens (P. Sineux 2007, p. 74-75). Ainsi Euripide aurait peut-être fait mention d'Amphiaraos afin de flatter les revendications athéniennes sur Oropos, et le tragique aurait mentionné la faculté empyromantique en se fondant sur une mauvaise lecture d'Hérodote qui liait le héros argien au sanctuaire d'Apollon Isménios à Thèbes.

(71) Si Thèbes l'emporte sur les Sept, les Épigones réussissent leur vengeance. P. Cloché 1952, p. 17-19; W.G.G. FORREST 1982, p. 288-294. 
précis de leur exposition en Béotie, il est possible (mais pas incontestable) qu'elles aient été tout de suite exposées chez Apollon Isménios. En tout cas, comme le souligne Roland Crahay ${ }^{72}$, cette mention d'Amphiaraos par Hérodote est «un élément étranger mal raccordé à l'ensemble du récit, simple aition de la présence d'offrandes lydiennes dans ce sanctuaire». Hérodote déclare que seule Delphes avait émis une réponse favorable lors de l'épreuve des oracles. Puis il ajoute incidemment Amphiaraos dont il ne connaît, par ailleurs, pas la réponse fournie (contrairement à celle de Delphes $)^{73}$. Ensuite, il décrit toutes les offrandes que Crésus a envoyées à Delphes afin de s'attirer

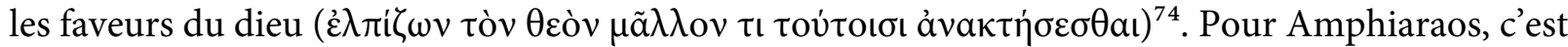
parce que Crésus a appris son «mérite et son malheur ( un bouclier et une lance en or $^{75}$. Roland Crahay précise encore, de son côté, que, lors de la deuxième consultation, Amphiaraos est encore associé à Delphes, mais que Crésus ne récompense plus que le sanctuaire d'Apollon (I, 53). Mieux, lors de la troisième visite, seul le sanctuaire de Delphes est mentionné (I, 55). Tout ceci pousse R. Crahay à penser que la mention d'Amphiaraos dans le récit d'Hérodote serait en réalité un ajout postérieur de la part de l'historien. Il l'aurait effectué parce que, ayant vu ces offrandes à Thèbes (et n'ayant pas obtenu d'explication quant à leur arrivée), il aurait établi un lien d'analogie entre celles-ci et le test des oracles ${ }^{76}$. À moins que la mention d'Amphiaraos ne provienne des informateurs béotiens d'Hérodote, qui conféraient à ces offrandes une origine lydienne, rehaussant ainsi le prestige du sanctuaire d'Amphiaraos. Plus simplement, il n'est pas exclu que les offrandes de Crésus à l'Amphiareion de Thèbes aient été déplacées dans le temple d'Apollon à la suite du déclin et de l'abandon de l'Amphiareion thébainn ${ }^{77}$.

Quoi qu'il en soit, il faut souligner que Crésus témoignait sa préférence pour Apollon: en atteste la liste des divers lieux et sanctuaires auxquels il aurait offert des présents autres que ceux mentionnés en étroite relation avec sa consultation des oracles. Parmi eux, trois sites sont à retenir: Delphes (qui reçoit un bouclier dans le temple d'Athéna Pronaia), le sanctuaire d'Apollon Isménios de Thèbes (où se trouve un trépied d'or, à distinguer des autres présents destinés à Amphiaraos), et, avant tout, le sanctuaire des Branchides de Milet (dont il nous est dit que les offrandes étaient semblables et égales en poids à celles de Delphes $)^{78}$.

Si l'on réexamine sous cet angle le récit d'Hérodote, il est clair que ce long exposé offre un prétexte ingénieux à l'auteur pour entamer une suite de digressions sur les événements qui se déroulent en Grèce continentale ${ }^{79}$.

\section{LA NATURE DE L'ALLIANCE ENTRE CRÉSUS ET LACÉDÉMONE}

Revenons aux propos de l'historien et attardons-nous sur cette phrase clef de I, 69 déjà citée

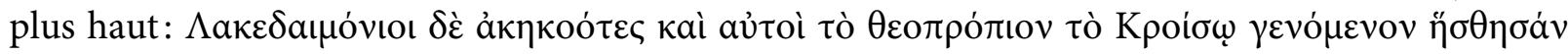

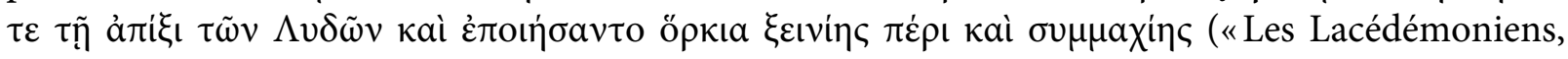
qui avaient eux aussi entendu parler de l'oracle rendu à Crésus [sur les plus puissants des Grecs],

(72) R. Crahay 1956, p. 196-197.

(73) HÉrodote, I, 48-49.

(74) HÉrodote, I, 50.

(75) Hérodote, I, 52. De même, la mention du héros qui selon la légende avait perçu la mort du petit Archémoros à Némée comme un signe funeste annonçant l'échec de l'expédition argienne contre Thèbes (comme Delphes annonçant la future fin de Crésus), pourrait provenir également d'une tragédie perdue traitant de la maison des Mermnades (voir note 37).

(76) R. Crahay 1956, p. 196-197.

(77) P. Roesch 1984, p. 176; R. Parker 2008, p. 151.

(78) HÉrodote, I, 92.

(79) J. A. S. Evans 1978-1979, p. 35 ; J. Griffin 2006, p. 54. 
se réjouirent de la venue des Lydiens et conclurent des serments d'hospitalité et d'alliance»).

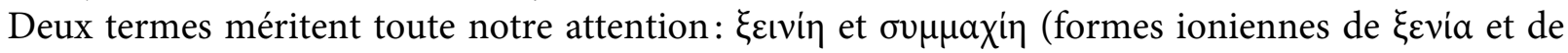

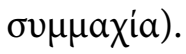

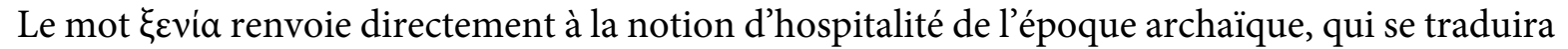
par $\varphi$ ı ía (amitié) à l'époque classique ${ }^{80}$. Ce vocable, couramment utilisé en diplomatie, exprime la relation d'amitié qu'entretiennent des communautés civiques, ou de puissantes familles, entre elles. De plus, comme tout traité implique une relation d'amitié, on est conduit à associer $\varphi \iota \lambda$ ía à

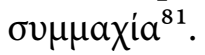

$\Sigma v \mu \mu a x i ́ a$ se réfère quant à lui à la notion d'alliance proprement dite. Ce terme désigne un état de fait, la nature de la relation qui lie deux ou plusieurs États à la suite d'un accord convenu entre $\mathrm{eux}^{82}$. Cependant, l'amitié doit être distinguée avec soin de l'alliance, car l'affirmation de la première n'implique pas ipso facto la seconde. Ce n'est pas parce que deux États se proclament «amis» qu'ils sont automatiquement des «alliés» ${ }^{83}$. De plus, comme le souligne D.J. Mosley:

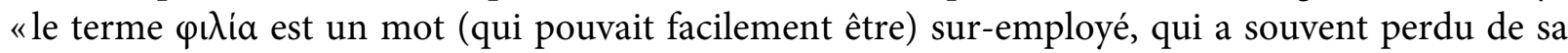
forcedans le cadre d'échanges diplomatiques; dans le meilleur des cas, il pouvait signifier une relation favorable (positive relationship) et dans le pire, il ne renvoyait à rien, ou rien de plus qu'un accord de non-agression ou de non-belligérance, entre deux parties ${ }^{84}$. Néanmoins, comme c'est le cas ici, une relation d'amitié supposait souvent une alliance ${ }^{85}$.

Ajoutons que $\sigma v \mu \mu a \chi i a$ renvoie à tout type alliance (offensive ou défensive), contrairement à

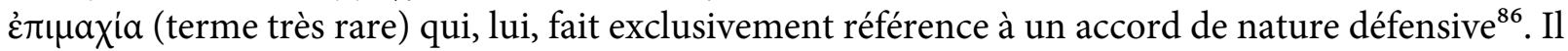
faut en tout cas observer que cette différenciation n'est attestée qu'au $\mathrm{V}^{\mathrm{e}}$ siècle. Avant l'émergence de cette distinction, et encore à la période durant laquelle écrit Hérodote (et même après), les

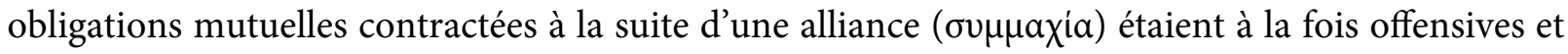
défensives ${ }^{87}$.

Cela étant posé, dans le cas qui nous préoccupe (en I, 69), Hérodote n'utilise pas d'entrée de jeu le terme de $\varphi \lambda \lambda i$ a mais bien celui de $\xi \varepsilon v i ́ a$ signifiant «l'hospitalité». Elle suppose une relation contraignante, qui implique que les voyageurs, messagers, ambassadeurs, et autres personnalités de passage doivent être reçus comme il se doit, selon certaines règles. Une fois accueilli, le visiteur acquiert une dimension sacrée: il devient comme «intouchable», tout entier sous la protection de son hôte qui devient garant de son intégrité physique ${ }^{88}$.

Cependant, Hérodote laisse entendre que les Spartiates étaient liés à Crésus par une alliance militaire $(\sigma v \mu \mu \alpha \chi i \alpha)$. En témoignent les préparatifs réalisés par Lacédémone afin de venir en aide au roi lydien assiégé. Cependant, cette mobilisation s'est avérée sans objet à la suite de la venue

(80) A. Giovannini 2007, p. 228.

(81) Idem, p. 228-230.

(82) Idem, p. 229.

(83) Par exemple: le traité entre Sybaris et ses voisins (dont les noms sont inconnus) entre 550 et 525; et le traité entre Rome et Carthage qui fut conclu 28 ans avant l'invasion de Xerxès en Grèce (Polybe, III, 22). - D. J. Bederman 2001, p. 159.

(84) Il donne plusieurs exemples (mais plus tardifs): l'alliance de 384 entre Athènes et Chios (qui veut préserver les accords de la Paix du Roi); l'alliance perpétuelle entre Athènes et Denys de Syracuse de 367 (qui n'est autre qu'un traité de non agression); etc. - F. AdCock et D. J. Mosley 1974, p. 208-209.

(85) Idem, p. 206-209.

(86) A. Giovannini 2007, p. 241-244. Le terme ne se rencontre pas avant Thucydide, qui mentionne une alliance

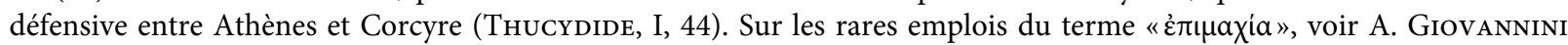
2007, p. 228.

(87) D. J. Bederman 2001, p. 162 ; F. Adcock et D. J. Mosley 1974, p. 122.

(88) Voir à ce sujet: F. Adcock et D. J. Mosley 1974, p. 164 sq.; D. J. Bederman 2001, p. 110 sq.; C. Gill et al. 1998; A. Giovannini 2007, p. 45 sq. 
d'un héraut lydien déclarant que Sardes venait d'être investie par les Perses ${ }^{89}$. En réalité, ce passage pourrait se révéler n'être qu'un élément autorisant le lien entre sa digression sur Sparte (avec sa guerre pour Thyréa) et son récit principal sur la chute de Sardes. Par ailleurs, Hérodote laisse entendre que la guerre entre Sparte et Argos était terminée lorsque le héraut lydien vint demander

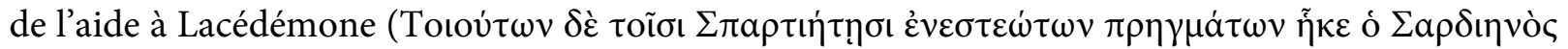

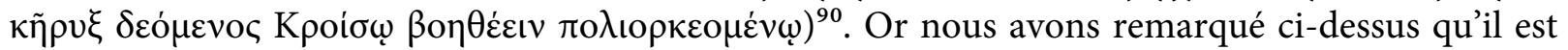
permis de dater le conflit entre ces deux cités de quelques années avant la chute de la capitale lydienne. De plus, il a été démontré que le rapprochement entre ces deux événements n'est qu'une approximation ultérieure. C'est pourquoi nous avons quelques raisons de penser que le passage sur les préparatifs spartiates afin de venir en aide à Crésus est un ajout de la part d'Hérodote qui tente de lier sa digression à son récit principal ${ }^{91}$.

Sur ces précisions, plusieurs scénarios s'offrent à nous:

1. Sparte n'aurait jamais conclu d'alliance militaire ( $\left.\sigma v \mu \mu \alpha \chi^{i \alpha}\right)$ de type offensif ou défensif avec Crésus. De plus, Sparte en acceptant de conclure une telle alliance, s'engageait à combattre au loin, ce qu'elle a souvent hésité à faire ${ }^{92}$. En effet, elle craignait un soulèvement des Hilotes et des Messéniens, voire une attaque d'Argos (en témoigne la bataille que cette dernière et Sparte se sont livrée au sujet de Thyréa $)^{93}$. Par contre, une relation de type $\xi \varepsilon v i ́ a$ n'est pas à exclure. Crésus aurait décidé de nouer des liens d'hospitalité avec Lacédémone à la suite de la victoirede celle-ci sur Argos, qui passait pour la plus puissance cité grecque du continent. Ainsi, dans une relation d'hospitalité, Crésus et Sparte auraient opéré à un échange de cadeaux (d'abord de l'or pour la statue d'Apollon du Thornax de la part du roi lydien; un cratère de bronze ensuite volé par les Samiens de la part de Lacédémone). Par conséquent, ces derniers se seraient retrouvés "alliés», sans connotation militaire ${ }^{94}$. Ainsi, ces deux États distants géographiquement nouaient tout simplement des liens entre eux. Pour entériner cette «alliance», les deux parties auraient envoyé leurs présents respectifs.

2. Sparte aurait accepté l'alliance militaire désirée par Crésus. En effet, celle-ci était la reconnaissance internationale de sa première place en Grèce à la suite de sa victoire récente sur sa rivale Argos, lors de la «bataille des champions», pour le contrôle de la Thyréatide. Lacédémone aurait donc accepté cette relation probablement dans le but de profiter des présents du riche roi lydien, en lui faisant miroiter une aide armée en cas de besoin tout en sachant que le risque d'y être contraint était inexistant. En effet, aux yeux des Grecs, Crésus passait pour un roi invincible sur le champ de bataille. Pour ces derniers, il était donc inimaginable que la Lydie tombe face à la puissance perse qui allait, de façon inattendue, succéder aux Mèdes. Dans ce cas, une alliance avec Crésus ne comportait que des avantages: à la fois une gloire sans pareille en pays grec, mais aussi des cadeaux généreux en métal

(89) HÉrodote, I, 83.

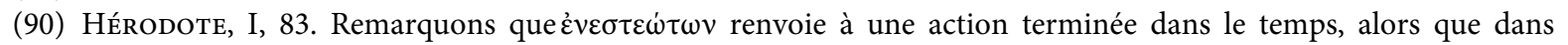

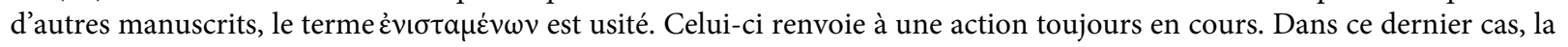
guerre entre Sparte et Argos ne était pas encore terminée.

(91) Sans oublier que dans l'esprit de l'historien, Crésus avait bien conclu une alliance de type militaire (en témoigne son usage du mot $\sigma v \mu \mu \alpha \chi i \alpha)$. Donc rajouter dans son récit un élément allant dans ce sens lui permettait d'établir un lien entre deux passages de son récit et un événement vraisemblable mais sans valeur historique.

(92) Sparte refusa d'aider les Ioniens lors des campagnes d'Harpage après la chute de Crésus (HÉrodote, I, 152) et lors de la révolte d’Ionie en 498 (HÉrodote, $\mathrm{V}, 50$ ).

(93) HÉrodote, I, 82-83.

(94) Car comme le souligne König, rapporté par Kaplan : à cette époque, il n’y avait pas de distinction entre une alliance

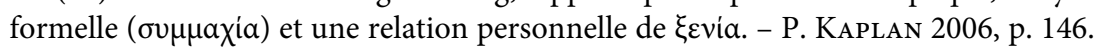


précieux. Tout cela sans l'envoi de troupes puisque le Mermnade n'en aurait sûrement pas eu besoin, capable de faire face au danger.

De ces hypothèses, on doit conclure qu'il faut placer la «bataille des champions» avant la chute de Sardes (contrairement à ce que laisse entendre Hérodote). De plus, on peut déduire que le souvenir de ces deux anciens événements, sans doute proches dans le temps, conduisit à les rapprocher, voire à les synchroniser comme dans d'autres cas bien connus. De notre côté nous préférons la première supposition qui semble la plus conforme aux traditions archaïques: Crésus n'aurait en réalité tissé que des liens d'hospitalité avec la cité de Sparte.

Revenons maintenant sur les rapports que Crésus entretenait plus spécialement avec la clef de sa diplomatie avec les Grecs, à savoir le sanctuaire de Delphes mais aussi les Delphiens ${ }^{95}$.

\section{LES RAPPORTS ENTRE CRÉSUS ET LES DELPHIENS}

À l'instar de la présence d'offrandes du roi lydien au sanctuaire, les rapports privilégiés noués entre la communauté des Delphiens et le Mermnade ne sont pas à mettre en doute. En effet, Hérodote déclare qu'en échange des offrandes et des cadeaux faits aux Delphiens ${ }^{96}$, ces derniers accordèrent à Crésus et aux Lydiens rien moins que, à tout jamais, la promantie ${ }^{97}$, l'atélie, la proédrie et le droit pour tout Lydien qui le désire de devenir Delphien ${ }^{98}$. Par ailleurs, on doit noter que ces échanges fondent une forte relation d'hospitalité entre Crésus (représentant des Lydiens) et la communauté des Delphiens. Cette dernière entraînait l'obligation d'accueillir, de protéger ainsi que de faire acte de bienveillance envers les ambassadeurs lydiens ${ }^{99}$. Ces honneurs accordés aux Lydiens semblent longtemps restés d'actualité. Effectivement, ils seront rappelés au $\mathrm{II}^{\mathrm{e}}$ siècle par les décrets delphiques pour Matrophanès de Sardes ${ }^{100}$. Ce dernier y renouvelle les «bons rapports» que les Delphiens ( $\Delta \varepsilon \lambda \varphi$ oĩ $)$ ont avec les gens de Sardes depuis les temps anciens ( $\tau \dot{\alpha} \tau \varepsilon$

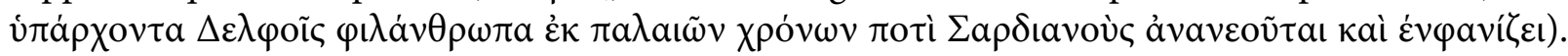
Matrophanès en appelle également à «la priorité pour consulter l'oracle qui leur avait été concédée

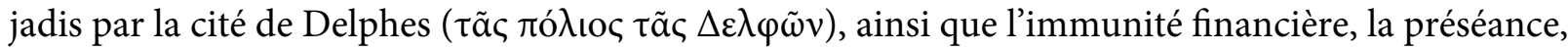

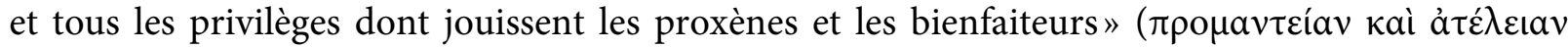

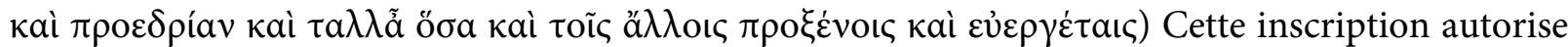
à authentifier les honneurs qu'avait reçus le roi lydien en échange de ses nombreuses offrandes et cadeaux envoyés à Delphes et aux Delphiens.

(95) Le sanctuaire de Delphes était administré depuis sans doute le début du $\mathrm{VI}^{\mathrm{e}}$ siècle (à la suite de la première guerre sacrée) par l'amphictionie pyléo-delphique, dont le conseil se réunissait deux fois par an. Son rôle était d'organiser les panégyries et les marchés, gérer les finances, protéger le sanctuaire et ceux qui le fréquentaient, surveiller le carrefour des voies commerciales et aussi la fonction d'agonothesia des Pythia. - P. SÁNCHEz 2001, p. 67-80.

(96) Crésus, satisfait de la réponse obtenue lors de sa deuxième consultation sur l'attitude qu'il devait adopter à l'égard des Perses, se serait enquis du nombre des Delphiens. Ainsi, il aurait fait don de deux statères d'or par homme ( $\pi \dot{\varepsilon} \mu \psi a \varsigma$

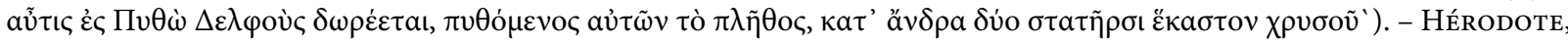
I, 54.

(97) Pierre Sánchez souligne que seuls les Delphiens avaient le droit d'octroyer ce privilège, et non les Amphictions. P. SÁNCHEZ 2001, p. 72.

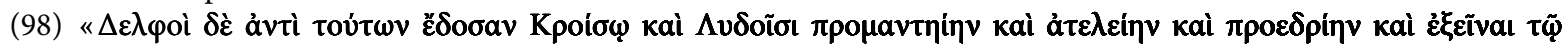

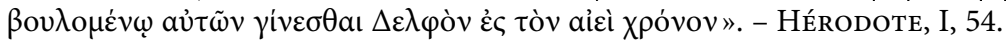

(99) A. Giovannini 2007, p. 45.

(100) Inscriptions reprises dans C. Michel 1900, p. 211 et 218. Elles sont traduites et commentées dans J. PouilLOux 1974, p. 159-169. Notons que le nom de Crésus n'apparaît pas dans cette inscription. 


\section{CONCLUSION}

Pour conclure, si l'historicité des oracles «anciens» invitant Crésus à s'allier avec les plus puissants des Grecs peut être mise en doute ${ }^{101}$, les relations diplomatiques que Crésus aurait entretenues avec la Grèce continentale sont moins incertaines. En effet, ses cadeaux et ses offrandes aux sanctuaires de Delphes, à Thèbes, ainsi qu'à Sparte lui ont permis d'éblouir les Grecs. Malgré ses pressions sur les puissantes communautés grecques d'Asie Mineure, il avait la volonté d'offrir à la Grèce une bonne entente.

Quant à la nature de l'alliance qui aurait uni Crésus à Lacédémone, plusieurs lectures s'offrent à nous. Ou bien Sparte n'a tissé que des liens d'hospitalité ( $\xi \varepsilon v i \alpha)$ avec le Mermnade, à la suite de sa victoire sur Argos pour le contrôle de la Thyréatide (forte de son statut de plus puissante parmi les Grecs): dans ce cas, cette relation impliquait qu'elle était tout de même «alliée » sans obligation militaire (les préparatifs spartiates n'étant qu'un élément permettant de lier sa digression sur la guerre entre Sparte et Argos avec son récit principal sur la chute de Sardes). Alors, leurs liens

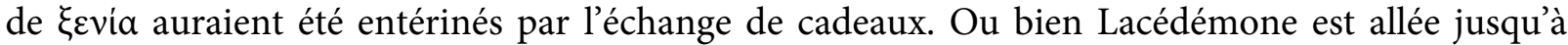
accepter l'alliance militaire ( $\sigma \nu \mu \mu \alpha \chi i$ ia) proposée par Crésus dans la foulée de sa victoire sur Argos. Celle-ci était alors le signe de reconnaissance internationale de son nouveau statut de cité la plus puissante de Grèce. Ainsi, elle profitait des largesses de Crésus sans imaginer qu'elle devrait un jour intervenir en Lydie, tant ce royaume paraissait invincible aux yeux des Grecs (en l'absence de concurrent crédible).

En ce qui concerne la nature des liens qui unissaient les Delphiens à Crésus, celle-ci pourrait fort bien avoir été une relation d'hospitalité, une entente qui aurait été établie lors du règne du dernier roi de Lydie $^{102}$, un lien qui aurait été rappelé quatre siècles plus tard, lors de la visite de Matrophanès ${ }^{103}$.

Kevin LELOUX

Département des sciences historiques - Université de Liège

\section{Bibliographie}

Adcock, F. et D. J. Mosley, 1974, Diplomacy in Ancient Greece, London, Thames \& Hudson.

Alty, J. H. M., 1982, «Dorians and Ionians», in JHS, 102, p. 1-14.

Asheri, D., A. Lloyd et A. Corcella, 2007, A commentary on Herodotus. Books I-IV, édité par O. Murray et A. Moreno avec la contribution de M. Brosius, Oxford, Oxford University Press.

Bederman, D. J., 2001, International law in Antiquity, Cambridge, Cambridge University Press.

Busine, A., 2002, Les Sept Sages de la Grèce antique: transmission et utilisation d'un patrimoine légendaire d'Hérodote à Plutarque, Paris, De Boccard.

Cloché, P., 1952, Thèbes de Béotie. Des origines à la conquête romaine, Louvain - Paris, Nauwlaerts - Desclée de Brouwer.

(101) La première place reconnue de Sparte ne pouvant être pour autant exclue après la «bataille des Champions» contre Argos.

(102) Voir déjà sous Alyatte, avant la première guerre sacrée et l'établissement de l'amphictionie, époque où le sanctuaire était administré par les seuls Delphiens.

(103) Mais rien ne dit que cette relation n'était plus d'actualité tout au long de ces siècles. 
Crahay, R., 1956, La littérature oraculaire chez Hérodote, Paris, Les Belles Lettres, (Collection de la Bibliothèque de la Faculté de Philosophie et Lettres de l'Université de Liège, fascicule 138).

Daux, G., 1957, «Remarques sur la composition du Conseil amphictionique», in Bulletin de correspondance hellénique, 81, p. 95-120.

Polignac, F. (de), 1995 [1984], La naissance de la cité grecque. Cultes, espace, et société VIII ${ }^{e}-V_{I I}{ }^{e}$ siècles avant J.-C., Paris, Éditions de la découverte.

Deshours, N., 1999, «Les Messéniens, le règlement des mystères et la consultation de l'oracle d'Apollon Pythéen à Argos", in Revue des Études Grecques, 112, p. 463-484.

Evans, J. A. S., 1978-1979, «What happened to Croesus?», in The Classical Journal, 74, p. 34-40.

Finley, M. I., 1982, Early Greece. The Bronze and the Archaic Age, New-York - London, Norton \& company.

Forrest, W.G.G., 1982, «Central Greece and Thessaly», in J. Boardman et N. G. L. Hammond (éd.), The Cambridge Ancient History, III-3, The Expansion of the Greek World, Eight to Sixth Centuries B.C., Cambridge, Cambridge University Press, p. 286-320.

Gill, C. et al., 1998, Reciprocity in Ancient Greece, Oxford, Oxford University Press.

Giovannini, A., 2007, Les relations entre États dans la Grèce antique du temps d'Homère à l'intervention romaine (ca 700-200 av. J.-C.), Stuttgart, Franz Steiner Verlag, (Historia, Einzelschriften, Heft 193).

Griffin, J., 2006, «Herodotus and Tragedy», in C. Dewald et J. Marincola (éd.), The Cambridge Companion to Herodotus, Cambridge, Cambridge University Press, p. 46-59.

Hall, J. M., 1999, Ethnic identity in Greek antiquity, Cambridge, Cambridge University Press.

Hammond, N.G.L., 1982, «The Peloponnese», in J. Boardman et N.G. L. Hammond (éd.), The Cambridge Ancient History, III-3, The Expansion of the Greek World, Eight to Sixth Centuries B.C., Cambridge, Cambridge University Press, p. 321-360.

Jeffery, L. H., 1988, «Greece before the Persian invasion», in J. Boardman, N. G. L. Hammond, D. M. Lewis, M. Ostwald (éd.), The Cambridge Ancient History, IV, Persia, Greece and Western Mediterranean c. 525 to 479 B.C., Cambridge, Cambridge University Press, p. 347-367.

Kaplan, P., 2006, «Dedications to Greek Sanctuaries by Foreign Kings», in Historia, 55-2, p. 129-152.

La Bua, V., 1977, «Gli Ioni e il conflitto lidio - persiano», in Miscellanea Greca e Romana, V, p. 1-64.

Lacroix, L., 1949, Les reproductions des statues sur les monnaies grecques. La statuaire archaïque et classique, Liège, Faculté de Philosophie et Lettres, (Collection de la Bibliothèque de la Faculté de Philosophie et Lettres de l'Université de Liège, fascicule 116).

Legrand 1932 (a), Hérodote. Histoires, I, Livre I: Clio, texte établi et traduit par P. E. Legrand, Paris, Les Belles Lettres.

-, 1932 (b), Hérodote. Histoires, Introduction, texte établi par P. E. Legrand, Paris, Les Belles Lettres (collection des Universités de France. Série grecque, 73).

Lenfant, D., 2011, «Hérodote d'Halicarnasse », in D. Lenfant (dir.), Les Perses vus par les Grecs. Lire les sources classiques sur l'Empire achéménide, Paris, Armand Collin, (Collection U), p. 214-227.

Liverani, M., 2003, «The Influence of Political Institutions on Trade in the Ancient Near East (Late Bronze to Early Iron Age)», in C. Zaccagnini (éd.), Mercanti e politica nel mondo antico, Roma, «l'Erma» di Bretschneider, p. 119-138.

Loraux, N., 1979, «L'autochtonie: une topique athénienne. Le mythe dans l'espace civique», in Annales. Economies, Sociétés, Civilisations, 34, p. 3-26.

—, 1996, Né de la Terre. Mythe et politique à Athènes, Paris, Seuil.

Mauss, M., 1923-1924, «Essai sur le don. Forme et raison de l'échange dans les sociétés archaïques», in Année sociologique, seconde série I, p. 30-186.

Michel, C., 1900, Recueil d'inscriptions grecques, Bruxelles, Lamertin.

Nafissi, M., 2009, «Sparta», in K. A. Raaflaub et H. Van Wees (éd.), A companion to Archaic Greece, Chichester, Wiley-Blackwell, p. 117-137. 
Parke, H. W. et D. E. W. Wormell, 1956, The Delphic oracle, volume I, The history, Oxford, Basil Blackwell.

Parker, R., 2008, «Religion and the Athenian Empire», in P. Low (éd.), The Athenian Empire, Edinburgh, Edinburgh University Press, (Edinburgh Reading On The Ancient World), p. 146-158.

Piérart, M., 1990, «Un oracle d'Apollon à Argos», in Kernos, 3, p. 319-333.

Pouilloux, J., 1974, «Les décrets delphiques pour Matrophanès de Sardes», in Bulletin de correspondance hellénique, 98, p. 159-169.

Richer, N., 1994, «Aspects des funérailles à Sparte», in Cahiers du Centre Gustave Glotz, 5, p. 51-96.

_, 2012, La religion des Spartiates. Croyances et cultes dans l'Antiquité, Paris, Les Belles Lettres.

Roesch, P., 1984, «l'Amphiaraion d'Oropos», in G. Roux (dir.), Temples et sanctuaires, Séminaire de recherche 1981-1983, Lyon, Maison de l'Orient et de la Méditerranée Jean Pouilloux, 1984 (Travaux de la maison de l'Orient), p. 173-184.

Saïd, S., 2002, «Herodotus and Tragedy», in E. J. Bakker, I. J. F. De Jong et H. Van Wees (éd.), Brill's Companion to Herodotus, Leiden-Boston-Köln, Brill, p. 117-147.

Sánchez, P., 2001, L'amphictionie des Pyles et de Delphes. Recherches sur son rôle historique, des origines au II siècle de notre ère, Stuttgart, Franz Steiner Verlag, 2001 (Historia, Einzelschriften, Heft 148).

Segal, C., 1971, "Croesus on the Pyre: Herodotus and Bacchylides», in Wiener Studien, 5 (1971), p. 39-51.

Sineux, P., 2007, Amphiaraos. Guerrier, devin et guérisseur, Paris, Les Belles Lettres, 2007 (La vérité des mythes).

Sordi, M., 1957, «La fondation du collège des naopes et le renouveau politique de l'Amphictionie au Iv siècle ", in Bulletin de correspondance hellénique, 81, p. 38-75.

Taplin, O., 1997, «The pictorial record», in P. E. Easterling (éd.), The Cambridge Companion To Greek Tragedy, Cambridge, Cambridge University Press, p. 69-90.

-, 2007, Pots \& Plays. Interactions between Tragedy and Greek Vase-painting of the Fourth Century B.C., Los Angeles, J.-Paul Getty.

Thomas, R., 2001, «Ethnicity, Genealogy, and Hellenism in Herodotus», in I. Malkin (éd.), Ancient Perception of Greek Ethnicity, Cambridge, Harvard University Press, (Center for Hellenic Studies Colloquia 5), p. 213-233.

Van Wees, H., 1998, «Reciprocity in Anthropological Theory», in C. Gill et alii (éds), Reciprocity in Ancient Greece, Oxford, Oxford University Press, p. 13-49.

Will, É., 1956, Doriens et Ioniens. Essai sur la valeur du critère ethnique appliqué à l'étude de l'histoire et de la civilisation grecques, Paris, Les Belles Lettres, 1956 (Publication de la faculté des lettres de l'Université de Strasbourg, fascicule 132). 
